\title{
PI Stabilization for Congestion Control of AQM Routers with Tuning Parameter Optimization
}

\author{
S.Chebli ${ }^{1}$, A.Elakkary ${ }^{1}$, N.Sefiani ${ }^{1}$, N.Elalami ${ }^{2}$ \\ ${ }^{1}$ LASTIMI, Graduate School of Technology of Salé, Crown Prince Street, B.P : 227 Salé medina-Morocco \\ ${ }^{2}$ Mohamdia School of Engineers, P.O. Box. 765, Ibn sina Street, Agdal Rabat Morocco
}

\begin{abstract}
In this paper, we consider the problem of stabilizing network using a new proportional- integral (PI) based congestion controller in active queue management (AQM) router; with appropriate model approximation in the first order delay systems, we seek a stability region of the controller by using the HermiteBiehler theorem, which isapplicable to quasipolynomials. A Genetic Algorithm technique is employed to derive optimal or near optimal PI controller parameters.
\end{abstract}

Keywords - Congestion Control, Activequeue Management (AQM), Stabilization, PI Controller, First Order Delay System,Genetic Algorithm (GA).

\section{INTRODUCTION}

$\mathrm{R}$ ECENTLY and due to the explosive growth of computer networks, several non linear phenomena of the internet have been discovered. For instance, it has been shown that network traffic congestion results in long time delays for data transmission and often makes the queue length in the buffer of the intermediate router overflow, and can even lead to network collapse. To face the congestion problems, TCP chiefly uses a network congestion avoidance which comprehend different aspects of an additiveincrease multiplicative-decrease (AIMD) scheme, with other schemes such as slow-start[1][2] and [3].

It was shown that the problem of congestion control and development of AQM could be considered as a problem of regulating. Thus, due to the limitations of packet-dropping probability and the effects of propagation delays in TCP networks, the TCP AQM network was modeled as a time-delayed system based on a fluid-based model of the dynamics of the TCP and RED, developed by the stochastic theory $[4][5][6]$ and [7].

From the control theory, the AQMs types PI [8], PID were built to control the congestion phenomenon. In this paper, we extend our previous work [9] by implementing Genetic Algorithm (GA) in determining PI Controller parameters to compensate the delay in the system. The Genetic Algorithm has been considered as a useful optimization techniques employing the principles of natural genetic systems [10] to search a global solution of the optimization problem.

\section{TCP/AQM MODEL}

Our study will focus on the sharing of a communication link between multiple transmitters at remote locations (Fig. 1).

In [6], the authors modeled TCP process with stochastic differential equations without taking slow start and timeoutmechanisms into account. In this model, the congestion window $\mathrm{w}(\mathrm{t})$ increase linearly if no packet loss is detected;otherwise it halves. Based on some reasonable assumptions, the following relations were gotten:

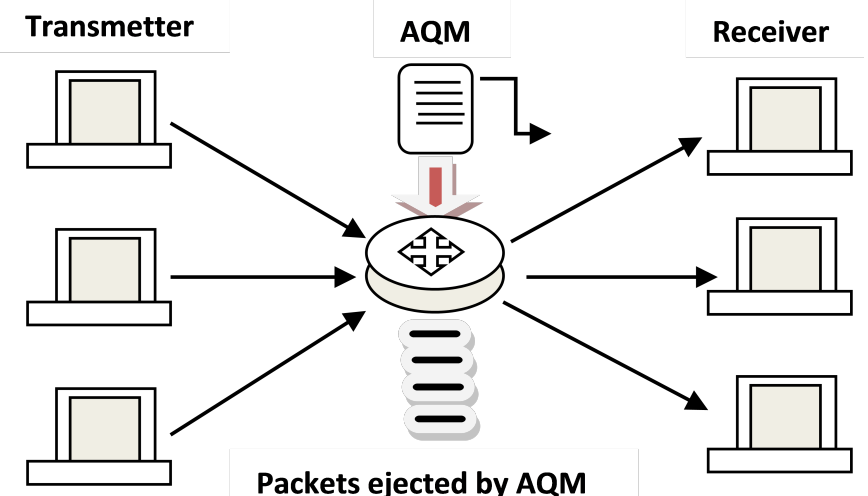

Fig. 1. Topology studied

$\left\{\begin{array}{l}\dot{w}(t)=\frac{1}{R(t)}-\frac{w(t) \cdot w(t-R(t))}{2 R(t-R(t))} p(t-R(t)) \\ \dot{q}(t)=\frac{w(t)}{R(t)} N(t)-C\end{array}\right.$

With $R(t)=\frac{q(t)}{C}+T p$, where $T p$ is the propagation delay, $q(t)$ is the queue length at the router, $C$ is the router's transmission capacity,

thus $\frac{q(t)}{C}$ is the queuing delay and $R(t)$ is the round trip time delay, $N(t)$ denotes the TCP load factor.

$\dot{w}(t)$ and $\dot{q}(t)$ denote the time-derivative of $W(t)$ and $q(t)$, respectively $p(t)$ is the probability of packet mark due to the AQM mechanism at the router.

The linearization of (1) and (2) about the operating point is carried out in (1) and the perturbed variables about the operating point satisfy

$$
\left\{\begin{array}{c}
\delta \dot{w}(t)=-\frac{N}{R_{0}^{2} C}\left(\delta w(t)+\delta w\left(t-R_{0}\right)\right) \\
-\frac{1}{R_{0}^{2} C}\left(\delta q(t)-\delta q\left(t-R_{0}\right)\right)-\frac{R_{0} C^{2}}{2 N^{2}} \delta p\left(t-R_{0}\right) \\
\delta \dot{q}(t)=\frac{N}{R_{0}} \delta w(t)-\frac{1}{R_{0}} \delta q(t)
\end{array}\right.
$$

From (3) and (4) we derive the transfer function from $\delta p$ to $\delta q$ :

$$
\frac{\delta q(s)}{\delta p(s)}=-\frac{R^{3} C^{3}}{2 N^{2}} \frac{A(s) e^{-R s}}{1+A(s) R s e^{-R s}}
$$

Where $A(s)=\frac{1}{\frac{R^{3} C}{N} s^{2}+\left(R+\frac{R^{2} C}{N}\right) s+2}$

Considering a negative feedback control system with the AQM being the controller, the system to be controlled is given by[9]: 


$$
\begin{aligned}
& G(s)=\frac{R^{3} C^{3}}{2 N^{2}} \frac{A(s) e^{-R s}}{1+A(s) R s e^{-R s}} \\
& =\frac{R^{3} C^{3}}{2 N^{2}} \frac{e^{-R s}}{\frac{R^{3} C}{N} s^{2}+\left(R+\frac{R^{2} C}{N}\right) s+2+R s e^{-R s}}
\end{aligned}
$$

\section{AQM FOR CONGESTION CONTROL}

A. Approximation of the control model of a first order delay system

In the process control industry, most systems can be roughly modeled as a first order system with delay:

$$
\hat{\mathrm{G}}(s)=\frac{K e^{-L s}}{T s+1}
$$

Where $\mathrm{K}, \mathrm{T}$ and $\mathrm{L}$ represent respectively the state gain, the constant time and the time delay of the plant. These three parameters are supposed to be positive.

We already carry out this approximation calculation in [9], we found:

$$
\left\{\begin{array}{c}
K=\frac{R^{3} C^{3}}{4 N^{2}} \\
T=R\left(\frac{R^{4} C^{2}}{4 N^{2}}+\frac{2 R C}{N}-\frac{2 R^{2} C}{N}+2\right)^{\frac{1}{2}} \\
L=\frac{R^{2} C}{2 N}+2 R-T
\end{array}\right.
$$

\section{B. Preliminary results}

Large number problems in process control engineering are related to the presence of delays. These delays are involved in the dynamic models whose equations characteristics are of the following form [12, 13]:

$$
\begin{aligned}
& \delta(\mathrm{s})=\mathrm{d}(\mathrm{s})+\mathrm{e}^{-\mathrm{L}_{1} \mathrm{~s}} \mathrm{n}_{1}(\mathrm{~s})+\mathrm{e}^{-\mathrm{L}_{2} \mathrm{~s}} \mathrm{n}_{2}(\mathrm{~s})+\cdots \\
& +e^{-L_{m} s} n_{m}(s)
\end{aligned}
$$

Where: $d(s)$ and $n(s)$ are polynomials with real coefficients and $\mathrm{Li}$ represent time delays. These characteristic equations are recognized as quasi-polynomials. We consider the case which meets the following assumptions:

$$
\begin{aligned}
& \text { A1: } 0<\mathrm{L}_{1}<\mathrm{L}_{2}<\cdots<\mathrm{L}_{\mathrm{m}} \\
& \text { A2: } \operatorname{desg}(\mathrm{d}(\mathrm{s}))=\mathrm{n} \text { and } \operatorname{deg}\left(\mathrm{n}_{\mathrm{i}}(\mathrm{s})\right)<\mathrm{n} \\
& i=1,2,3, \ldots, m
\end{aligned}
$$

One can consider the quasi-polynomials $\delta^{*}(\mathrm{~s})$ described by:

$$
\begin{aligned}
& \delta^{*}(\mathrm{~s})=\mathrm{e}^{\mathrm{L}_{\mathrm{m}} \mathrm{s}} \delta(\mathrm{s})
\end{aligned}
$$

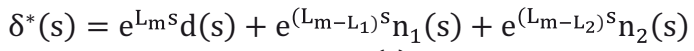

$$
\begin{aligned}
& +\cdots+\mathrm{n}_{\mathrm{m}}(\mathrm{s})
\end{aligned}
$$

The zeros of $\delta(\mathrm{s})$ are identical to those of $\delta^{*}(\mathrm{~s})$ since $\mathrm{e}^{\mathrm{L}_{\mathrm{m}} \mathrm{s}}$ does not have any finite zeros in the complex plan. However, the quasipolynomial $\delta^{*}(\mathrm{~s})$ has a principal term since the coefficient of the term containing the highest powers of $\mathrm{s}$ and $\mathrm{e}^{\mathrm{s}}$ is nonzero. If $\delta^{*}(\mathrm{~s})$ does not have a principal term, then it has an infinity roots with positive real parts [15].

The stability of the system with characteristic equation (13) is equivalent to the condition that all the zeros of $\delta^{*}(\mathrm{~s})$ must be in the open left half of the complex plan. We said that $\delta^{*}(\mathrm{~s})$ is Hurwitz or is stable. The following theorem gives a necessary and sufficient condition for the stability of $\delta^{*}(\mathrm{~s})$.

Theorem 1[11][12][13][14][15]

Let $\delta^{*}(s)$ be given by (3) and write:

$$
\delta^{*}(j \omega)=\delta_{r}(\omega)+j \delta_{i}
$$

Where $\delta_{r}(\omega)$ and $\delta_{i}(\omega)$ represent respectively the real and imaginary parts of $\delta^{*}(j \omega)$. Under conditions (A1) and $(A 2), \delta^{*}(s)$ is stable if and only if:

1: $\delta_{r}(\omega)$ and $\delta_{r}(\omega)$ have only simple, real roots and these interlace,

2: $\delta_{i}^{\prime}\left(\omega_{0}\right) \delta_{r}\left(\omega_{0}\right)-\delta_{i}\left(\omega_{0}\right) \delta_{r}^{\prime}\left(\omega_{0}\right)>0$ for some $\omega_{0}$ in $[-\infty,+\infty]$ Where $\delta_{r}^{\prime}(\omega)$ and $\delta_{i}^{\prime}(\omega)$ denote the first derivative with respect to $\omega$ of $\delta_{r}(\omega)$ and $\delta_{i}(\omega)$, respectively.

A crucial stage in the application of the precedent theorem is to verify that and have only real roots. Such a property can be checked while using the following theorem.

Theorem 2 [8][12][13][14][15]

Let $M$ and $N$ designate the highest powers of $s$ and $e^{s}$ which appear in $\delta^{*}(s)$. Let $\eta$ be an appropriate constant such that the coefficient of terms of highest degree in $\delta_{r}(\omega)$ and $\delta_{i}(\omega)$ do not vanish at $\omega=\eta$. Then a necessary and sufficient condition that $\delta_{r}(\omega)$ and $\delta_{i}(\omega)$ have only real roots is that in each of the intervals $-2 l \pi+\eta<\omega<2 l \pi+\eta, l=l_{0}, l_{0}+1, l_{0}+2 \ldots$

$\delta_{r}(\omega)$ or $\delta_{i}(\omega)$ have exactly $4 l N+M$ real roots for a sufficiently large $l_{0}$.

\section{Stabilization using PI Controller}

We consider now the feedback control system shownin Fig. 2, where $r$ is the command signal, $y$ is the output of the plant, $\hat{G}(s)$ is the plant to be controlled, and C(s) is the controller.

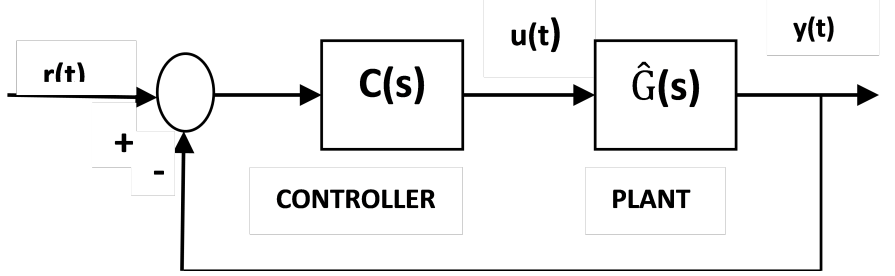

Fig. 2. Feedback control system.

We will focus on the case when the controller is the PI controller described by the following transferfunction:

$$
\mathrm{C}(\mathrm{s})=\mathrm{K}_{\mathrm{p}}+\frac{\mathrm{K}_{\mathrm{i}}}{\mathrm{s}}
$$

The objective is to determine the set of controller parameters $(\mathrm{Kp}$, Ki) for which the closed-loop system is stable.

\section{Theorem 3 [11][12][13][14][15]}

The range of $K_{p}$ value, for which a solution to PI stabilization problem for a given stable open-loop plant exists, is given by:

$$
-\frac{1}{K}<K_{p}<\frac{T}{K L} \sqrt{\alpha_{1}^{2}+\frac{L^{2}}{T^{2}}}
$$


Where $\alpha_{1}$ the solution of the equation $\tan (\alpha)=-\frac{T}{L} \alpha$ in the interval $\left[\frac{\pi}{2}, \pi\right]$

The proof of this theorem is detailed in[9].

To determine the stabilizing PI parameters for a first order delayed system, analgorithm has been proposed deduced from the theorems above [9].

\section{Genetic Algorithm Optimization}

GeneticAlgorithms (GAs) are adaptive heuristic search algorithm based on the evolutionary ideas of natural selection and genetics. As such they represent an intelligent exploitation of a random search used to solve optimization problems. The main idea in this technique is derived from natural evolution so there are some biological operators such as Crossover, Mutation and Selection [16]. The evolution from one generation to the following is based on the use of the three operators; selection, crossover and mutation which are applied to all the elements of the population. There are three part of this process which are randomly generated, namely the Initial Population, the Crossover and the Mutation. In the first step of the process, the Initial Population in the Genetic Algorithm generates some random solutions. In the second step, the random value of Crossover aid to make new offspring and in the third step, with random value of Mutation let to change a few of gens [17][22][23].

The principle of regulator parameters optimization by the Genetic Algorithms is shown by Fig. 3. It is about the search of parameters $k_{p}$ and $\mathrm{k}_{\mathrm{i}}$ in the area of stability.

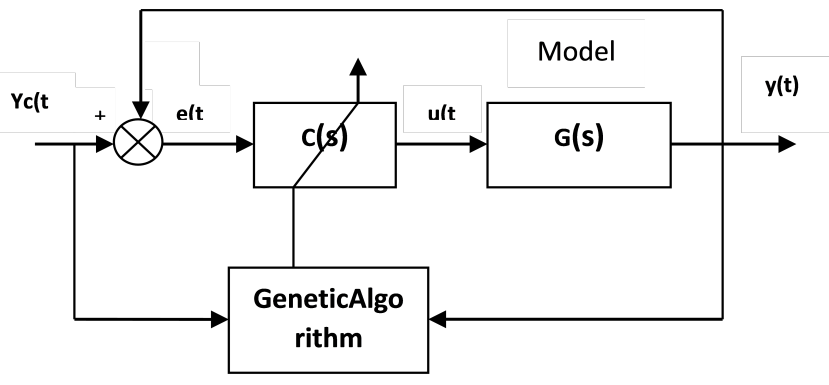

Fig. 3. The optimization principle by genetic algorithm.

\section{A. The objective functions fitness values}

The most crucial step in applying GA is to choose the objective functions that are used to evaluate fitness of each chromosome. Some works, like [18] and [19] use performance indices as the objective functions. The both authors use Integral of Time multiplied by Absolute Error (ITAE), Integral of Absolute Magnitude of the Error (IAE), and Integral of the Squared Error (ISE). Here we use all three performance indices stated above and Integral of Time multiplied by the Squared Error (ITSE) to minimize the error signal e(s) and compare them to find the most suitable one. The performance indices are defined as follow:

$$
\begin{aligned}
& I S E=\sum_{0}^{t_{\max }} e(t)^{2}, \quad I A E=\sum_{0}^{t_{\max }}|e(t)| \\
& I T A E=\sum_{0}^{t_{\max }} t|e(t)|, \quad I T S E=\sum_{0}^{t_{\max }} t e(t)^{2}
\end{aligned}
$$

Where $e(t)$ is the error signal in time domain.

If we want to minimize the tuning energy, the ITAE criteria and the
IAE are considered. In the case where we privilege the rise time, we take the ITSE criterion. In order to guarantee the tuning energetic cost, we choose the ISE criterion.

The calculation steps of the control law are summarized by the following algorithm [20]:

1. Introduction of the following parameters:

- $\max _{\text {pop }}$ individuals number by population.

- initial population.

- gen $_{\max }$ generation number.

2. Initialization of the generation counter $($ gen $=1)$.

3. Initialization of the individual counter $(j=1)$.

4. For $t=1 s$ to $t=t_{\max }$ efficiency evaluation of $j^{t h}$ population individual.

5. Individual counter incrementing $(j=j+1)$.

- If $j<\max _{\text {pop }}$, going back to step 4 .

- If not: application of the genetic operators (selection, crossover, mutation) for founding a newpopulation.

6. Generation counter incrementing gen $=g e n+1$,

7. If $g e n<g e n_{\max }$; going back to step 3 .

8. Taking $K_{p}$ opt and $K_{i}$ opt which correspond to the best individual in the last population (individual making the best fitness).

On the following, the genetic algorithm is characterized by generation number equal to $100, \mathrm{Pc}=0.8, \mathrm{Pm}=0.08$ and individual number by population equivalent to 50 .

We now present an example that illustrates the application of the results presented in this section.

\section{Simulation Results}

The performance of the closed-loop system with the new PI controller is tested by simulation. The number of TCP flows is taken to be $\mathrm{N}=60$, the link capacity $\mathrm{C}=3750$ packets/sec, round trip time $\mathrm{R}=$ $400 \mathrm{~s}$, the desired queue size $q_{0}=150$ packets, and the buffer size $q_{\max }$ $=200$ packets [9].

We obtain the stability region in Kp, Ki plane, presented in Fig. 4:

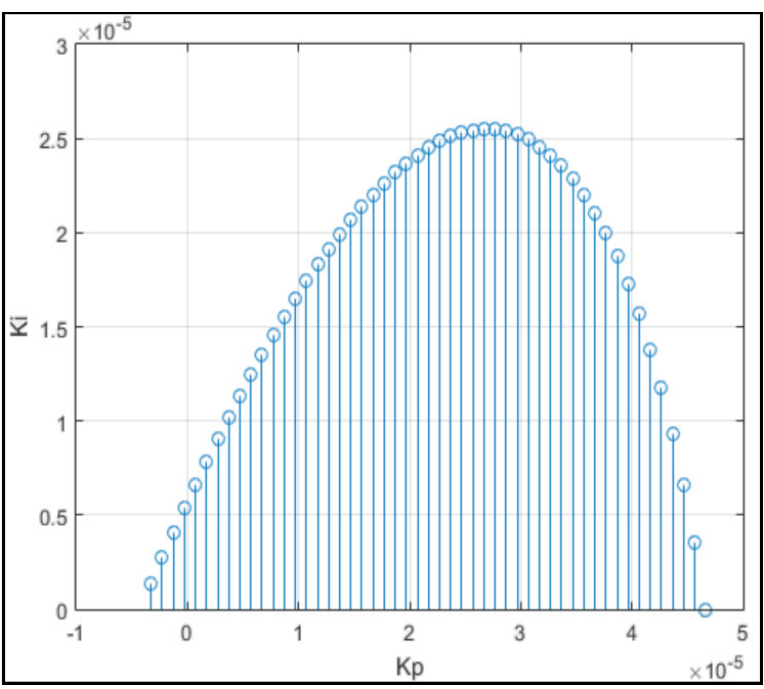

Fig. 4. Stability region of the system

The closed loop step responses of PI conroller optimum parameters supplied by Genetic Algorithm are illustrated in the following Fig. 5: 


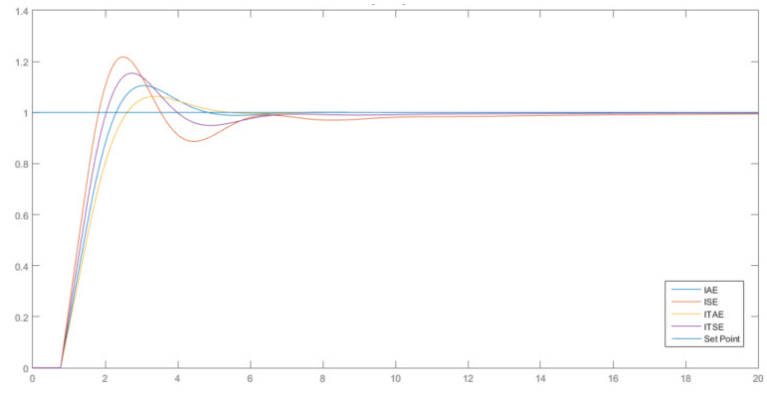

Fig. 5. Step response for the different optimal PI controllers.

The performances are rise time, settling time and overshoot. The aim of the controllers is to minimize the transient state response performances. Numerical values of these performances of the closed loops are given in Table 1 for comparison:

TABLE 1:

NUMERICAL VALUES OF STANDARD PERFORMANCE MEASURES.

\begin{tabular}{|l|c|c|c|c|}
\hline \multicolumn{1}{|c|}{ Criterion } & ISE & IAE & ITAE & ITSE \\
\hline $\boldsymbol{K}_{\boldsymbol{p}}$ opt $\left(10^{-4}\right)$ & 0.22 & 0.1642 & 0.1489 & 0.0296 \\
\hline $\boldsymbol{K}_{\boldsymbol{i}}$ opt $\left(10^{-4}\right)$ & 0.0293 & 0.0325 & 0.0296 & 0.0321 \\
\hline Rise Time (s) & 0.8183 & 1.1453 & 1.3229 & 0.9630 \\
\hline Settling Time (s) & 9.7619 & 4.4026 & 4.6105 & 6.1405 \\
\hline Overshot (\%) & 21.7792 & 10.5320 & 6.3206 & 15.4224 \\
\hline
\end{tabular}

\section{A. Standard performance measures:}

It can be seen from Table 1 that GA optimized by the four criterions provides a very small rise time which is beneficial for the congestion control of the AQM routers, chiefly the one obtained by the ISE criterion. The best value of the settling time is provided by IAE and ITAE and that of the overshoot is given by GA optimized by ITAE.

\section{CONCLUSION}

Congestion is one of the most important problems faced in communications networks. In this paper we proposed to solve this problem by applying a new approach to compute the stability region for first order delay system controlled by PI controllers. This result is based on an extension of the Hermite-Biehler Theorem to quasipolynomials. Then, we were interested in search of optimal PI for performance criteria, inside the stability region by resorting to the Genetic Algorithm techniques as a method of optimization.

Finally, it is pointed out that the effectiveness of the proposed approach has been verified via simulation.

\section{REFERENCES}

[1] V. Jacobson, "Congestion avoidance and control," in Proceedings of ACM SIGCOMM, Stanford, CA, August 1988.

[2] D. Chiu and R. Jain, "Analysis of the increase/decrease algorithms for congestion avoidance in computer networks," Journal of Computer Networks and ISDN, vol. 17, no. 1, pp. 1-14, June 1989.

[3] Ohsaki H., Sugiyama K. and Imase M., Congestion Propagation among Routers with TCP Flows, International Journal of Computer Networks \& Communications, vol. 1, no. 2,pp. 112-127. 2009

[4] V. Misra, W. Gong, and D. Towsley. Stochastic differential equation modeling and analysis of TCP windowsize behavior. Technical Report ECE-TR-CCS-99-10-01, University of Massachusetts, October 1999.

[5] H. I. Alshimaa, A. EL-Sayed \& Z. Elsaghir, I. Z. Morsi, "Enhanced Random Early Detection (ENRED)" International Journal of Computer Applications (0975 - 8887) Vol. 92 - No.9, April 2014.

[6] V. Misra, W. Gong, and D Towsley. Fluid-based analysis of a network of AQM routers supporting TCP flows with an application to RED. In ACM
SIGCOMM, pages 151-160, August 2000.

[7] H. S. Low, F. Paganini, and J.C. Doyle. Internet congestion control.IEEE Control Systems Magazine, 22:28-43, Feb 2002.

[8] G. J. Silva, A. Datta and S. P. Bhattacharyya, "PI stabilization of first-orde systems with time-delay", Automatica, 37(12) :2025-2031, 2001.

[9] S. Chebli, A. Elakkary, N. Sefiani, N. Elalami, "New PI stabilization of first-order congestion control of active queue management routers", in Proceeding of Electrical and Information Technologies (ICEIT) Conference, pp.12 - 217, Marrakech, Morocco, March, 2015.

[10] Goldberg, D. E. Genetic algorithm in search, optimization, and machine learning, reading. MA: Addison Wesley, 1989.

[11] G. J. Silva, A. Datta and S. P. Bhattacharyya, "PID controllers for time delay systems", Birkhäuser, MA, Boston, 2001

[12] G. J. Silva, A. Datta and S. P. Bhattacharyya, Stabilization of Time Delay Systems, Proceedings of the American Control Conference, pp.963-970, 2000.

[13] G. J. Silva, A. Datta and S. P. Bhattacharyya, Stabilization of First-order Systems with Time Delay using the PID controller, Proceedings of the American Control Conference, pp. 4650-4655, 2001.

[14] G. J. Silva, A. Datta and S. P. Bhattacharyya, New synthesis of PID controller, IEEE transactions on automatic control, vol.47,No.2, 2002.

[15] G.J. Silva, A. Datta, S.P. Bhattacharyya, PID controllers for time delay systems, Springer, London, 2005.

[16] M. Martínez, S.García-Nieto, J.Sanchis, X.BlascoFerragud: Genetic algorithms optimization for normalized normal constraint method under Pareto construction. Advances in Engineering Software (AES) 40(4):260-267 (2009).

[17] G. Ye, Z. Tao, "Improved genetic algorithms based on chaotic mutation operation and its application". Multimedia Technology (ICMT), 2010 International Conference on 1-3 (2010).

[18] Ian Griffin, On-line PID Controller Tuning using Genetic Algorithms, Dublin City University, 2003.

[19] T. O'Mahony\& CJ. Downing (Cork Institute of Technology, Ireland), KlaudiuszFatla (Wroclaw University of Technology, Poland), Genetic Algorithms for PID Parameter Optimization, Minimizing Error Criteria.

[20] R. Farkh, K. Laabidi, M. Ksouri, "PI control of second order delay system with tuning parameter optimization", International Journal of Electrical and Electronics Engineering, vol.3, pp. 1-7, 2009.

[21] Zaldaña, H. R., and E. Castañeda, "The Use of Genetic Algorithms in UV Disinfection of Drinking Water", International Journal of Interactive Multimedia and Artificial Intelligence, 3(3), pp. 43-48, 2015

[22] Arranz, Á., and M. Alvar, "GPGPU Implementation of a Genetic Algorithm for Stereo Refinement", International Journal of Interactive Multimedia and Artificial Intelligence, 2(3), pp. 69-76, 2015

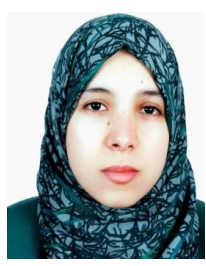

S. Chebli was born in Ouezzane, Morocco, in 1990. She is a PhD Student at Graduate School of Technology Salé/ Mohammadia Engineers School Rabat, in Automatic Engineering. She obtained the Engineer degree in Electromechanical Engineering from the National School of Mineral Industry of Rabat in July, 2013. Her research interests are in control systems, optimization methods, and network theories.

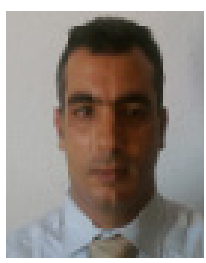

Ahmed El Akkary was born in Settat in Morocco, assistant professor at the Graduate School of Technology in Sale Morocco, winner of ENSET Rabat in 1995, in 2003 he obtained his DESA specialty automatic and manufacturing, then, in 2009, he obtained his doctorat from the Mohammadia School of Engineers of Rabat, specialty Automatic, researcher at the laboratory LASTIMI of EST Sale Morocco. His research interests are in control systems.

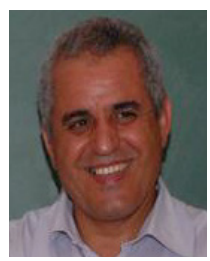

Mr Noureddine El Alami received his Engineering in Automatic and Industrial Computing from Mohammadia School of Engineering, Morocco, in 1980. He holds a degree in Applied Mathematics from the Faculty of Sciences, Rabat, Morocco. In 1986, he received his Doctor of Philosohy in Automatic from the university of Perpignann, France. He was a professor at Mohammadia School of Engineering, Morocco, in Applied Mathematics, Optimal Control, Non linear Systems and Signal Theory. His rechearch interests have revolved around the control of non-linear systems and their applications in engineering. He is the author or co-author of some papers in international conferences and journals. 